\title{
On the Generators of the Group of Units Modulo a Prime and Its Analytic and Probabilistic Views
}

\author{
Ricky B. Villeta ${ }^{1 *}$, Elmer C. Castillano ${ }^{2}$, and Roberto N. Padua ${ }^{3}$ \\ 1,2,3 University of Science and Technology of Southern Philippines, Cagayan de Oro City, Philippines \\ 1 https://orcid.org/0000-0001-8386-6798 \\ 2 https://orcid.org/0000-0002-1230-8817 \\ 3 https://orcid.org/0000-0002-2054-0835 \\ *Email Correspondence: rbvilleta35@usjr.edu.ph
}

\section{Abstract}

This paper further investigates the cyclic group $\left(Z_{p}\right)^{*}$ with respect to the primitive roots or generators $g \in\left(Z_{p}\right)^{*}$. The simulation algorithm that determines the generators and the number of generators, $g$ of $\left(Z_{p}\right)^{*}$ for a prime $p$ is illustrated using Python programming. The probability of getting a generator $\mathrm{g}$ of $\left(Z_{p}\right)^{*}$, denoted by $\frac{\phi(\phi(p))}{\phi(p)}$, is generated for prime $p$ between 0 to 3000. The scatterplot is also shown that depicts the data points on the probability $\frac{\phi(\phi(p))}{\phi(p)}$ of the group of units $\left(Z_{p}\right)^{*}$ with respect to the order $p-1$ of $\left(Z_{p}\right)^{*}$ for prime $p$ between 0 to 3000 . The scatterplot results reveal that the probability of getting a generator of the group of units $\left(Z_{p}\right)^{*}$ is fluctuating within the probability range of 0.20 to 0.50 , for prime $p$ modulus from 3 to 3000 . These findings suggest that the proportion of the number of generators of the group of units modulo a prime of order $p$ - 1, though fluctuating, is bounded from $20 \%$ to $50 \%$ for prime p modulus from 3 to 3000 .

Keywords: Group of units modulo a prime, $\left(Z_{p}\right)$;, primitive roots or generators of $\left(Z_{p}\right)^{*}$, simulation algorithm, probability of getting a generatorg of $\left(Z_{p}\right)^{*}$.

\subsection{Introduction}

Let $Z_{n}$ be the set of integers $\{0,1,2, \ldots, n-1\}$ under addition modulo $n$. Then the set of all elements $a$ of $Z_{n}$ relatively prime to $n$, that is, $\operatorname{gcd}(a, n)=1$, under multiplication modulo $n$ forms a group denoted by $\left(Z_{n}\right)^{*}$. The order of this group, $\left|\left(Z_{n}\right)^{*}\right|$, is equal to $\phi(n)$ where:

$$
\phi(n)=n \prod_{p / n}\left(1-\frac{1}{p}\right) .
$$

The function $\phi$ is called the Euler Totient function (Vinogradov, 2003).

The group $\left(Z_{n}\right)^{*}$ is cyclic if and only if $n$ is equal to $1,2,4, p^{k}$ or $2 p^{k}$ (Gauss, 1966). When $n=$ $p$ is prime, it follows that $\left(Z_{n}\right)^{*}$ is a cyclic group of 
order $\phi(p)=p-1$.

A number $\mathrm{g}$ is a generator of a cyclic group under multiplication modulo $n$, if for each $b$ in this group, there exists a $k$, such that $g^{k} \equiv b(\operatorname{modulo} n), \operatorname{gcd}(b, n)=1$. Such a generator is called a primitive root modulo $n$. The integer $k$ is called the index of $b$ to the base $g$ modulo $n$ (sometimes referred to as the discrete logarithm of $b$ to the base g modulo $n$ ). When $n=p$ is a prime, the number of primitive roots modulo $n$ is $\phi(\phi(p))=\phi(p-1)$, since a cyclic group of $p$ - 1 elements has $\phi(p-1)$ generators (Vinogradov, 2003). Knuth (1998) showed that:

$$
\frac{n}{\phi(n-1)}=O(\log \log n)
$$

so that for large $n$, the generators are very common among $\{2,3, \ldots, n-1\}$.

This study endeavors to investigate further the cyclic group $\left(Z_{p}\right)^{*}$, and the elements of $\left(Z_{p}\right)^{*}$, specifically the generators $g \in\left(Z_{p}\right)^{*}$ . The simulation algorithm that determines the generators and the number of generators, $\mathrm{g}$ of $\left(Z_{p}\right)^{*}$ for a prime $p$ is illustrated using the Python programming. The distribution of the resulting number of generators for each prime $p$ as modulus of the cyclic group $\left(Z_{p}\right)^{*}$ is presented using a scatterplot diagram. The probability of getting a generator $\mathrm{g}$ of $\left(Z_{p}\right)^{*}$, denoted by $\frac{\phi(\phi(p))}{\phi(p)}$ is also generated for prime $p$ between 1 and 3000 .
2.0 Prime Generators of $\left(Z_{p}\right)$ *

The group $\left(Z_{p}\right)^{*}$ under modulo $p$ is cyclic with $\phi(p)=p-1$ elements. The number of generators of this cyclic group, therefore is, at most $\phi(\phi(p))=\phi(p-1)$ (Vinogradov, 2003). We enumerated facts about the generators of $\left(Z_{p}\right)^{*}$ and had proven some of them. Wilson's Theorem (Burton, 2007, p. 94) in number theory is an important tool in deriving a result for the product of generators $g_{i}$ of $\left(Z_{p}\right)^{*}$ for a prime $p$. It says:

Theorem 2.1 (Wilson) Let $p$ be a prime number. Then $(p-1) ! \equiv-1$ modulo $p$.

While Wilson's result can be used as a primality test, however, it is computationally intractable. It remains an important theoretical result. Next, if $p$ is a prime, then $\left(Z_{p}\right)^{*}$ has $\phi(p)=p-1$ elements. Since $\left(Z_{p}\right)^{*}$ is cyclic, it has $\phi(p-1)$ generators.

\section{Examples 2.2}

(1) If $\mathrm{p}=11,\left(Z_{11}\right)^{*}$ has $\phi(11)=10$ elements and it has $\phi(10)=\phi(\phi(11))$ generators, that is, $\phi(10)=4$. The generators are $\{2,6,7,8\}$. Note that $2 \cdot 6 \cdot 7 \cdot 8 \equiv 1(\bmod 11)$ since $2 \cdot 6 \equiv 1(\bmod 11)$ and $7 \cdot 8=56 \equiv 1(\bmod 11)$.

(2) If $\mathrm{p}=17,\left(Z_{17}\right)^{*}$ has $\phi(17)=16$ elements, and it has $\phi(\phi(17))=\phi(16)=8$ generators, namely, $\{3,5,6,7,10,11,12,14\}$. We can re-group generators as follows $\{(3,6),(5,7)$, $(10,12),(11,14)\}$, so that $\prod_{i=1}^{8} g_{i} \equiv 1(\bmod 17)$. 
The following result shows that the product of generators $g_{i}$ of the group of units modulo a prime $p$ is congruent to 1 modulo $p$. Fermat's Theorem (Burton, 2007, p. 88) is used to prove this result.

Theorem 2.3 (Fermat's Theorem) Let $p$ be a prime and suppose that $p$ does not divide $a$. Then, $a^{p-1} \equiv 1(\bmod p)$.

Theorem 2.4 Let $p$ be a prime. Then $\left(Z_{p}\right)^{*}$ has $\phi(p-1)$ generators and

$$
\prod_{i=1}^{\phi(p-1)} g_{i} \equiv 1(\bmod p) .
$$

Proof: The first part follows from the fact that $\left(Z_{p}\right)^{*}$ has $\phi(p)=p-1$ elements. Since $\left(Z_{p}\right)^{*}$ is cyclic then, it has $\phi(p-1)$ generators. Next, take a generator $g_{k}$. By Fermat's Theorem (Theorem 2.3),

$$
g_{k}^{p-1} \equiv 1(\bmod p) \text { for } k=1,2, \cdots, \phi(p-1) .
$$

For each $j, g_{j}=g_{k}^{d_{j}}$ since $g_{k}$ is a generator. Now,

$$
\begin{aligned}
\prod_{j=1}^{\phi(p-1)} g_{j} & =\prod_{j=1}^{\phi(p-1)} g_{k}^{d_{j}}=g_{k}^{d_{1}} g_{k}^{d_{2}} \ldots g_{k}^{d_{\phi(p-1)}} \\
& =g_{k}^{d_{1}+d_{2}+\cdots+d_{\phi(p-1)}}=g_{k}^{\sum_{j=1}^{\phi(p-1)} d_{j}} .
\end{aligned}
$$

We can pair each term by their inverses and this gives:

$$
\prod_{i=1}^{\phi(p-1)} g_{i}=g_{k}^{\frac{\phi(p-1)}{2}(\phi(p))} \equiv 1(\bmod p)
$$

Consider, next, the primefactors of $\phi(p)$ where $p$ is a prime. Suppose that $\phi(p)=2 p_{1} p_{2} \cdots p_{k}$. Let $\mathrm{Q}$ be the set of all primes less than or equal to $p, Q=\left\{q_{1}, q_{2}, \cdots, q_{m}\right\}$. Then, it is clear that $\left\{p_{1}, p_{2}, \cdots, p_{k}\right\} \subseteq Q \subseteq\left(Z_{p}\right)^{*}$.
Lemma 2.5 Let Q be the set of all primes less than or equal to $p$ and let $P$ be the set of all prime factors of $\phi(p)$. Then $P \subseteq Q \subseteq\left(Z_{p}\right)^{*}$.

Proof: Let $p_{j} \in P$, then $p_{j} / \phi(p)$ and so $p_{j}<p$. Moreover, $\operatorname{gcd}\left(p_{j}, p\right)=1$, hence, $p_{j} \in Q \subseteq\left(Z_{p}\right)^{*}$. It follows that $P \subseteq Q$.

3.0 Analytic and Probabilistic Procedure in Finding Generators of the Cyclic Group, $\left(Z_{p}\right)^{*}$

An element of the group of units modulo a prime $p, g \in\left(Z_{p}\right)^{*}$ is a generator if $\left(Z_{p}\right)^{*}=\left\{g^{k}: k \in Z\right\}$. The computation of generators of the cyclic group, $\left(Z_{p}\right)^{*}$ is indispensable in pseudorandom number generators, error detecting codes, and in many cryptosystems such as the following: DiffieHellman key exchange protocol; ElGamal and Massey-Omura public key ciphers; DSA ElGamal and Nyberg-Rueppel digital signature (Adamski \& Nowakowski, 2015).

The following result, Theorem 3.1, Adamski \& Nowakowski (2015), in algebraic number theory is useful in the simulation algorithm which can be used to obtain the generators of the cyclic group, $\left(Z_{p}\right)^{*}$ modulo a prime $p$

Theorem 3.1 Let $\left(Z_{p}\right)^{*}$ be the cyclic group of the group of units modulo a prime $p$ of order $\phi(p)=p-1$. Let $2 p_{1} \cdot p_{2} \cdots p_{k}$ be the prime factorization of $\phi(p)$. Then, $g \in\left(Z_{p}\right)^{*}$ is a generator of $\left(Z_{p}\right)^{*}$ if and only if for all $i=1,2, \ldots, k$, $g^{\frac{\phi(p)}{p_{i}}}$ is not congruent to 1 modulo $p$. 


\section{Simulation Algorithm for Finding Generators of the Group of Units Modulo a Prime}

This section determines the simulation algorithm that constructs a large prime $p$ for the modulus of $\left(Z_{p}\right)^{*}$, and finds the generator and the number of generators of $\left(Z_{p}\right)^{*}$. Python programming was used in the implementation of this algorithm.

\section{Constructing the Large Prime $\boldsymbol{p}$ for the Modulus} of $\left(Z_{p}\right)^{*}$

In constructing the large prime $\mathrm{p}$ for the modulus of $\left(Z_{p}\right)^{*}$, the Miller-Rabin Test (Rabin, 1980) for the test of primality can be used.

\section{The Miller-Rabin Test of Primality}

Suppose $n$ is prime with $n>2$, hence $n$ 1 is even, which can be written as $2^{t} e$, where $t$ and $e$ are positive integers ( $e$ is odd). For each integer $x, 1<x<n$, then either $x^{e} \equiv \pm 1(\bmod n)$ or $x^{2^{r} e} \equiv-1(\bmod n)$ for any $r$ with $1 \leq r \leq t-1$.

The Miller-Rabin primality test is the contrapositive of the preceding statement, that is, in the event that we can find an $x^{e}$ is not congruent to 1 or $-1(\bmod n)$ or $x^{2^{r} e}$ is not congruent to -1 $\bmod (n)$, for all $1 \leq r \leq t-1$, then $n$ is not prime.

Finding the Generators $g \in\left(Z_{p}\right)^{*}$ for a Prime $\boldsymbol{p}$

The following outlines the simulation algorithm for finding the generators $g \in\left(Z_{p}\right)^{*}$ for a large prime $p$ as the modulus of $\left(Z_{p}\right)^{*}$ :

1. Determine the number $n$ if prime using the
Miller-Rabin primality test. If $n$ is prime, then denote it by $p$;

2. Get the prime factors of $p-1$, that is, $\phi(p)=p-1=2 p_{1} \cdot p_{2} \cdots p_{k}$

3. Initialize the list of generator;

4. Iterate $j$ from 1 to $\phi(p)=p-1$, the order or size of $\left(Z_{p}\right)^{*}$;

5. In every iteration $j$, initialize flag to a generator;

6. Iterate $i$ for all the prime factors of $\phi(p)=p-1$;

7. If $j^{\left(\frac{p-1}{i}\right)} \equiv 1(\bmod p)$, then make a flag that $j$ is not a generator;

8. Outside the iteration of the prime factors, provide a condition for checking the flag;

9. If flag is true, then $j$ is a generator and append to the list of generators of $\left(Z_{p}\right)^{*}$;

10. Count the number of generators of $\left(Z_{p}\right)^{*}$ in the list; and

11. Iterate steps 1 to 10 to generate all the generators of $\left(Z_{p}\right)^{*}$, for prime $p$ between 1 and 3000 .

4.0 Simulation Results for the Generators and Number of Generators of the Group of Units Modulo a Prime for Prime Modulus Between 0 to 3000

Figures 1, 2, 3, 4 and 5 depict the scatterplot for the data points on the number of generators of the group of units $\left(Z_{p}\right)^{*}$ versus the corresponding prime number modulus from 0 to 3000 . 


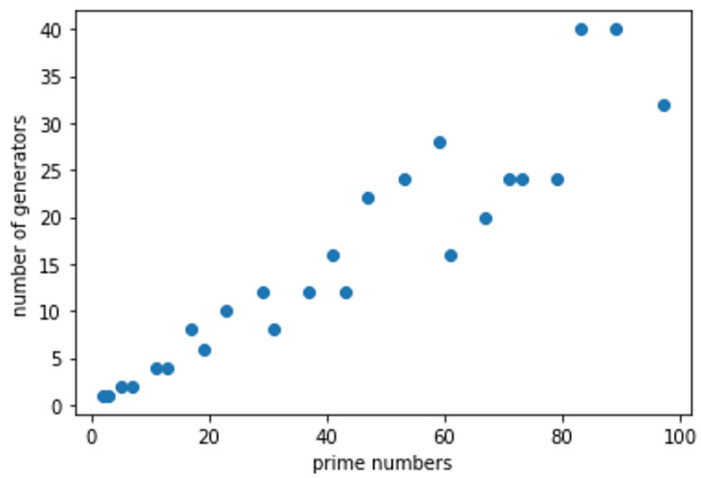

Figure 1. Scatterplot for the number of generators of $\left(Z_{p}\right)$ *versus the corresponding prime number modulus between 0 and 100

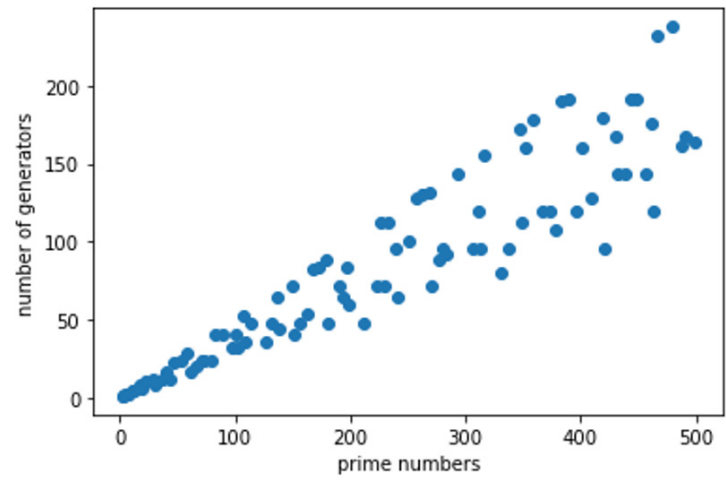

Figure 2. Scatterplot for the number of generators of $\left(Z_{p}\right)^{*}$ versus the corresponding prime number modulus between 0 and 500

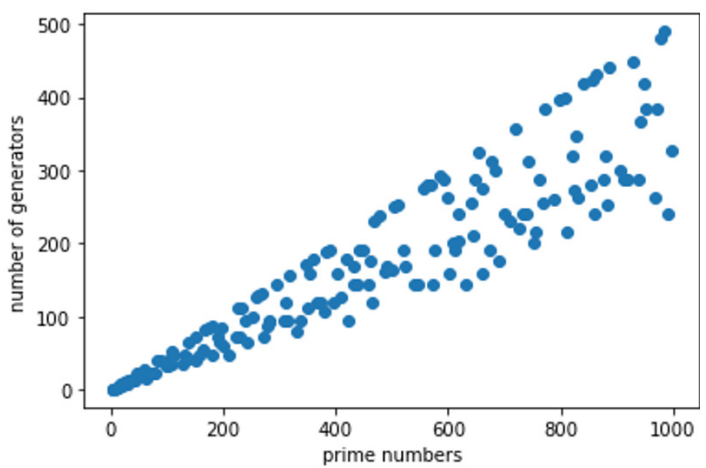

Figure 3. Scatterplot for the number of generators of $\left(Z_{p}\right)$ *versus the corresponding prime number modulus between 0 and 1000

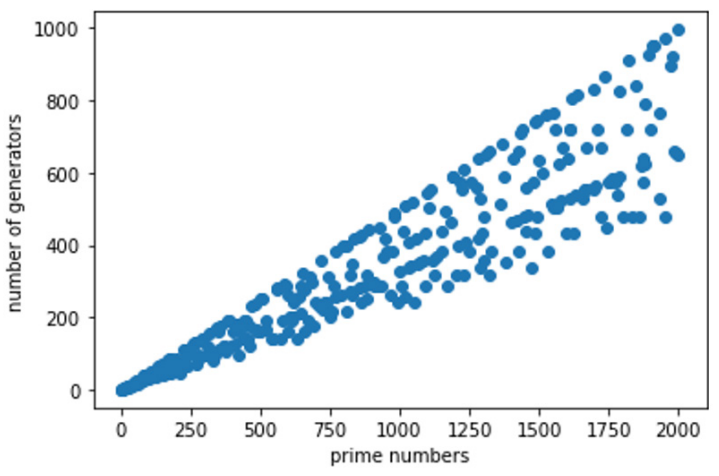

Figure 4. Scatterplot for the number of generators of $\left(Z_{p}\right)^{*}$ versus the corresponding prime number modulus between 0 and 2000

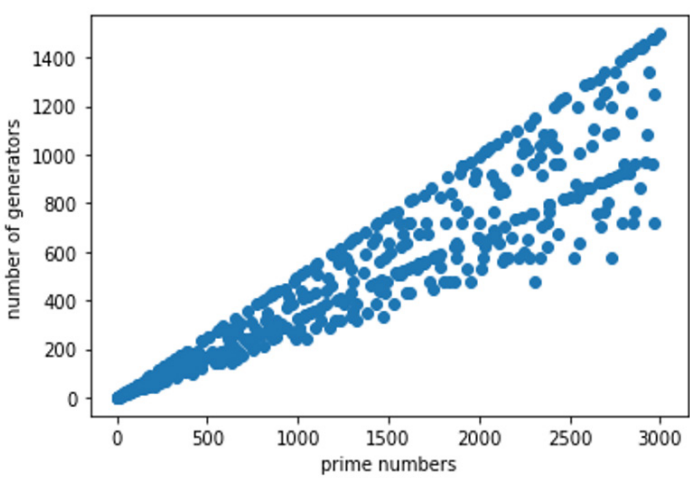

Figure 5. Scatterplot for the number of generators of $\left(Z_{p}\right)^{*}$ versus the corresponding prime number modulus between 0 and 3000

5.0 The Probability, $\frac{\phi(\phi(p))}{\phi(p)}$ Behavior of Finding a Generator of the Group of Units Modulo a Prime $\boldsymbol{p}$ for each Prime Modulus Between 0 to 3000

Figures $6,7,8,9$ and 10 depict the scatterplot for the data points on the probability $\frac{\phi(\phi(p))}{\phi(p)}$ of the group of units $\left(Z_{p}\right)^{*}$ versus the corresponding order $p-1$ of $\left(Z_{p}\right)^{*}$ for prime $p$ between 2 to 3000 . The scatterplot results reveal that the probability of getting a generator of the group of units $\left(Z_{p}\right)^{*}$ 
is fluctuating within the probability range of 0.20 to 0.50 , for prime $p$ modulus from 3 to 3000 . These findings suggest that the proportion of the number of generators of the group of units modulo a prime of order $p-1$, though fluctuating, is bounded from $20 \%$ to $50 \%$ for prime $p$ modulus from 3 to 3000 .

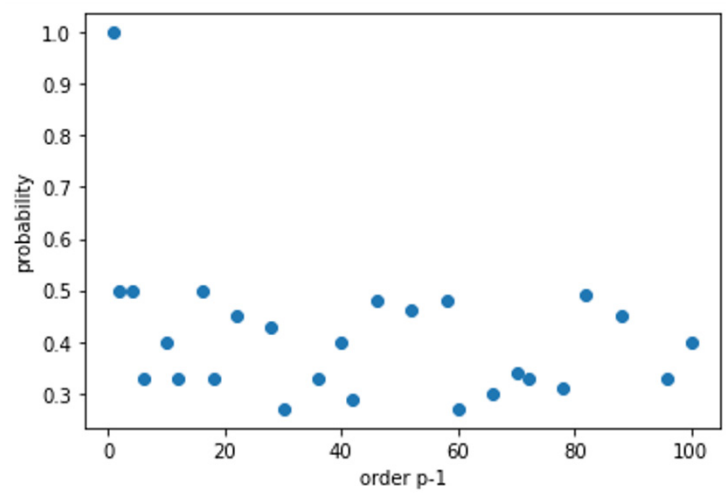

Figure 6. Scatterplot for the probability $\frac{\phi(\phi(p))}{\phi(p)}$ of the group of units $\left(Z_{p}\right)^{*}$ versus the corresponding order $p-1$ of $\left(Z_{p}\right)^{*}$ for prime $p$ between 0 and 100

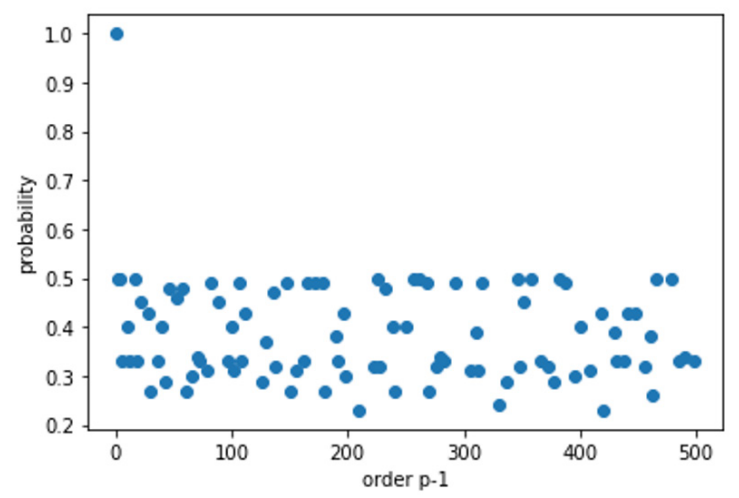

Figure 7. Scatterplot for the probability $\frac{\phi(\phi(p))}{\phi(p)}$ of the group of units $\left(Z_{p}\right)^{*}$ versus the corresponding order $p-1$ of $\left(Z_{p}\right)^{*}$ for prime $p$ between 0 and 500

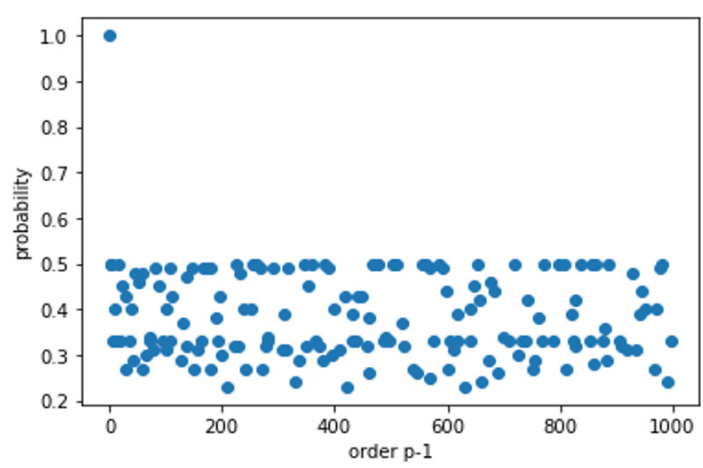

Figure 8. Scatterplot for the probability $\frac{\phi(\phi(p))}{\phi(p)}$ of the group of units $\left(Z_{p}\right)^{*}$ versus the corresponding order $p-1$ of $\left(Z_{p}\right)^{*}$ for prime $p$ between 0 and 1000

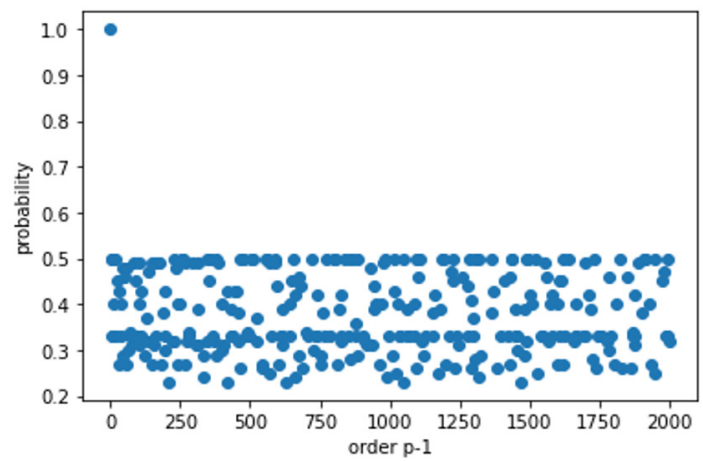

Figure 9. Scatterplot for the probability $\frac{\phi(\phi(p))}{\phi(p)}$ of the group of units $\left(Z_{p}\right) *$ versus the corresponding order $p-1$ of $\left(Z_{p}\right)^{*}$ for prime $p$ between 0 and 2000

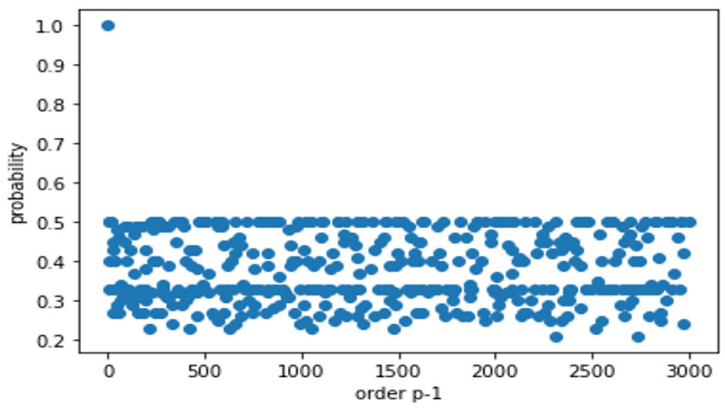

Figure 10. Scatterplot for the probability $\frac{\phi(\phi(p))}{\phi(p)}$ of the group of units $\left(Z_{p}\right)^{*}$ versus the corresponding order $p-1$ of $\left(Z_{p}\right)^{*}$ for prime $p$ between 0 and 3000 


\subsection{Conclusion}

This study investigated further the cyclic group $\left(Z_{p}\right) *$ with respect to the primitive roots or generators $g \in\left(Z_{p}\right)^{*}$. The simulation algorithm that determines the generators and the number of generators, $g$ of the cyclic group $\left(Z_{p}\right)^{*}$, for prime $p$ is illustrated using the Python programming. The probability of getting a generator $g$ of $\left(Z_{p}\right)^{*}$ denoted by $\frac{\phi(\phi(p))}{\phi(p)}$ is generated for prime $p$ between 0 to 3000 . The scatterplot results for the data points on the probability $\frac{\phi(\phi(p))}{\phi(p)}$ of the group of units $\left(Z_{p}\right)^{*}$ with respect to the order $p-1$ of $\left(Z_{p}\right)^{*}$ reveal that the probability of getting a generator of the group of units $\left(Z_{p}\right)^{*}$ is fluctuating within the probability range of 0.20 to 0.50 for prime $p$ modulus from 3 to 3000 . These findings suggest that the proportion of the number of generators of the group of units modulo a prime of order $p-1$, though fluctuating, is bounded from $20 \%$ to $50 \%$ for prime $p$ modulus from 3 to 3000 .

\section{References}

Adamski, T., \& Nowakowski, W. (2015). The average time complexity of probabilistic algorithms for finding generators in finite cyclic groups. Bulletin of the Polish Academy of Sciences,Technical Sciences, 63(4), 989-996. https://doi.org/10.1515/bpasts-2015-0112.

Burton, D. M., (2007). Elementary number theory (6thed.). McGraw-Hill.
Gauss, C. F. (1966). Disquisitiones arithmeticae (English ed). Springer-Verlag. https://doi. org/10.1007/978-1-4939-7560-0.

Knuth, D.E. (1998). The artofcomputerprogramming: Vol. 2. Seminumerical algorithms (3rd ed.). Addison-Wesley.

Rabin, M. O. (1980). Probabilistic algorithm for testing primality. Journal of Number Theory, 12(1), 128-138. https://doi.org/10.1016/0022 314X(80)90084-0.

Vinogradov, I. M. (2003). Elements of number theory. Dover Publications Inc. https://books.google. com.ph/books?id=xllfdGPM9t4C\&printsec $=f r$ ontcover\#v=onepage \&q\&f=false 